\title{
2 Innovation in der Peripherie und regionale Wissensbasen
}

Bei der Analyse von Innovation in ländlich-peripheren Regionen stellt sich zunächst die Frage nach der Abgrenzung. In der Wissenschaft und auch in Planungsdokumenten wird diese Abgrenzung häufig über statische Indikatoren wie die geographische Erreichbarkeit oder die Bevölkerungsdichte vorgenommen. Ein vereinfachendes Peripherieverständnis findet sich beispielsweise auch im aktuellen Österreichischen Raumentwicklungskonzept von 2011 (Humer 2018). Seit einigen Jahren gewinnt der Peripherisierungsdiskurs (KüHN und WECK 2012; KüHN 2015) an Einfluss, der auch die Grundlage für die vorliegende Studie darstellt. Dieses Kapitel geht also zunächst auf diesen Ansatz ein und skizziert in der Folge den aktuellen Forschungsstand zu Innovation in der Peripherie. Anschließend wird das konzeptionelle Framework vorgestellt, das die Grundlage für die quantitative Analyse einerseits und die qualitativen Interviews andererseits bildet.

\subsection{Von der Kern-Peripherie-Dichotomie zur Peripherisierung}

Frühe theoretische Ansätze in der Wirtschaftsgeographie, die bis ins 18. Jahrhundert zurückgehen, sehen vor allem in den mit der Distanz zu Zentren steigenden Transportkosten den entscheidenden Faktor für die Abgrenzung der Peripherie. Eine schwache wirtschaftliche Aktivität ergibt sich folglich daraus, dass es sich schlicht nicht lohnt, Produkte in abgelegenen Regionen zu produzieren, weil sie durch den Transport zu den Absatzmärkten zu teuer werden (Copus 2001). Auch die Polarisierungstheorien (MYRDAL 1957; HiRSCHMAN 1958) der 1950er Jahre sowie die New Economic Geography von Krugman (1991) basieren im Wesentlichen auf dieser Annahme.

Vor dem Hintergrund einer verbesserten Transportinfrastruktur und moderner Kommunikationstechnologie gelten diese Ansätze heute als überholt. Eine Kern-Peripherie-Abgrenzung rein auf Basis von räumlichen Distanzen und Agglomerationsvorteilen ist nicht mehr ausreichend, auch wenn dies immer noch in manchen wissenschaftlichen Studien und Dokumenten aus der Planungspraxis der Fall ist (DANSON und DE SoUZA 2012; LoRENTZEN 2012; KÜHN 2015). Als einer der ersten spricht CopUs (2001) von einer a-räumlichen Peripherie und unterstreicht die (mangelnde) Verfügbarkeit von leistungsfähiger IT-Infrastruktur, Humankapital und Netzwerken als entscheidende Größen.

In der Folge beschreibt KüHN (2015, S. 374) - basierend auf CRONE (2012, S. 50ff) - fünf Faktoren, die demnach für diese Abgrenzungsfrage zentral sind. Erstens sind Zentrum und Peripherie ein relationales Konzept, eine Region kann nur in Gegensatz zu einem Kern als peripher definiert werden. Zweitens ist der Ansatz prozessorientiert, 
zentral sind also Veränderungen. Drittens handelt es sich um ein multidimensionales Phänomen, es geht also nicht nur um räumliche, sondern auch demographische, ökonomische und politische Einflussfaktoren. Viertens spielt der Maßstab eine Rolle, da Regionen auf einer räumlichen Ebene zentral und auf einer anderen peripher sein können. Und fünftens handelt es sich bei Peripherie um ein dynamisches Konzept. Regionen können peripher oder zentral werden, etwa, wenn sich die wirtschaftlichen Rahmenbedingungen ändern.

Vor allem der letzte Punkt stellt dabei die Grundlage für eine aktive Regionalpolitik dar. Ohne ein dynamisches Verständnis sind Fortschritte in einer Region und eine Verbesserung ihrer Position nicht denkbar (LORENTZEN 2012). Dennoch sind statische Planungskonzepte weit verbreitet, weshalb die Autorinnen und Autoren zunehmend den Begriff der Peripherie zugunsten von Peripherisierung ablehnen. Letzterer deutet stärker auf die dynamische Komponente des Konzepts hin und wird damit der Realität besser gerecht (KüHN 2015). Eine zielführende, proaktive Regionalpolitik ist dabei nach wie vor von Bedeutung, da eine mangelnde Qualität auf politischer Ebene die Innovationstätigkeit von Regionen maßgeblich verringert (RoDRÍGUEZ-PosE und DI Cataldo 2015).

Zusammenfassend lässt sich also sagen, dass Peripherisierung zwar ein komplexeres, aber auch ein zielführendes Konzept im Gegensatz zum klassischen Kern-Peripherie-Verständnis ist. Eine räumliche Abgrenzung von zentralen und peripheren Regionen sollte also nicht nur auf Basis der geographischen Erreichbarkeit erfolgen, sondern auch nach Möglichkeit demographische, ökonomische und politische Faktoren miteinbeziehen. Zumindest einige dieser Faktoren sollten eine Entwicklung abbilden, also die Veränderung über die Zeit zeigen, um dem dynamischen Verständnis des Konzepts gerecht zu werden. Und schließlich sollte klar definiert werden, auf welcher Maßstabsebene und in Relation zu welchen Kernregionen die Analyse erfolgt. Die vorliegende Studie folgt deshalb methodisch bei der Einteilung in zentrale und periphere Regionen den Grundsätzen des Peripherisierungsdiskurses.

\subsection{Regionale Wissensbasen}

Neben demographischen, ökonomischen und politischen Faktoren - also beispielsweise einer Abwanderung von Personen im Erwerbsalter, dem Verlust von Arbeitsplätzen oder der Absenz von politischen Institutionen - spielt auch die Abkopplung von Wissensnetzwerken im Peripherisierungsdiskurs eine wichtige Rolle. In der modernen Wissensgesellschaft ist es auch für periphere Regionen von zunehmender Bedeutung, in diese globalen Netzwerke eingebunden zu sein (KüHN und WECK 2012; KüHN 2015). Während bisherige Arbeiten häufig nur darauf eingehen, ob diese wissensintensiven Branchen in einer Region vorhanden sind oder nicht, analysiert diese Studie die unterschiedlichen Voraussetzungen für Innovationen im Detail, indem der Ansatz der regionalen Wissensbasen berücksichtigt wird (ASHEIM und COENEN 2005; AsHeIm 2007). 
Diese Wissensbasen unterscheiden zwischen verschiedenen Voraussetzungen für Innovationen und geben deshalb darüber Auskunft, wie regionale Wissensnetzwerke ausgestaltet sind. Mittlerweile werden drei Basen unterschieden:

\section{Die Analytische Wissensbasis}

Analytisches Wissen ist häufig in Branchen zu finden, in denen Grundlagenforschung eine wichtige Rolle spielt, wie beispielsweise in den Lebenswissenschaften oder in der Informations- und Kommunikationstechnologie (IKT). Die Wissensgenerierung ist formalisiert, erfolgt also anhand branchenüblicher Standards und Firmen kooperieren häufig mit Universitäten. Das entstandene Wissen wird meist über Publikationen oder Patente verbreitet, folglich ist implizites Wissen - also ein Erfahrungswissen - von geringerer Bedeutung. Die analytische Basis erfordert Abstraktion, Theoriebildung und Hypothesenprüfung, weshalb die in den F\&E-Abteilungen beschäftigten Personen in der Regel über einen Universitätsabschluss und Forschungserfahrung verfügen. Der Fokus auf Grundlagenforschung führt oft zu radikalen Innovationen und folglich neuen Unternehmen, beispielsweise Startups und Spin-offs (AsHeIM und GerTLER 2005; AsHeim und Coenen 2006; Malmberg und Power 2006; Asheim 2007).

\section{Die Synthetische Wissensbasis}

Synthetisches Wissen ist in Innovationsprozessen vorhanden, in denen bestehendes Wissen auf innovative Weise neu kombiniert wird. Diese Wissensbasis lässt sich folglich häufig im produzierenden Gewerbe finden, in dem Innovationen oft in enger Abstimmung mit Kunden und Zulieferern zustande kommen. Gemeinsame Projekte mit Universitäten sind vorhanden, jedoch selten. Implizites Wissen, also Erfahrungswissen zu den Abläufen und Produkteigenschaften ist hingegen zentral und entsteht induktiv. Eine Ausbildung am Arbeitsplatz wie etwa Lehrberufe sind somit häufiger unter der Belegschaft in den F\&E-Abteilungen als wissenschaftliche Abschlüsse. Folglich sind inkrementelle Innovationen gängiger, Ausgründungen wie Spin-Offs kommen nur manchmal vor (Asheim und Gertler 2005; Asheim und Coenen 2006; MALMBerg und Power 2006; ASHeIM 2007).

\section{Die Symbolische Wissensbasis}

Dieses Wissen stellt eine Erweiterung des ursprünglichen Konzepts dar und ist vor allem eine Reaktion auf die zunehmende Bedeutung kreativer Branchen wie Werbung und Design. Innovation beruht auf neuen Ideen, die allerdings ein tiefgehendes Verständnis von Normen und Gewohnheiten in der betreffenden Branche und Region erfordern. Implizites Wissen spielt folglich eine wesentliche Rolle, ebenso Erfahrung, persönliche Fähigkeiten und Netzwerke, währende akademische Abschlüsse tendenziell unbedeutend sind (AsHEIM et al. 2007a; AsHEIM et al. 2007b).

Bei diesen Wissensbasen handelt es sich um idealtypische Formen, die sich in der Realität häufig überlappen. Ebenso beruhen unterschiedliche Phasen im Innovations- 
prozess auf unterschiedlichen Wissensbasen, so kann die Produktentwicklung hauptsächlich auf synthetisches Wissen zurückgreifen, während bei der Vermarktung der symbolischen Wissensbasis häufig die Schlüsselrolle zukommt (STRAMBACH und KLEMENT 2012; MANNICHE et al. 2017). Auch zeigt sich, dass Firmen besonders innovativ sind und ein hohes Wachstum aufweisen, wenn sie möglichst alle Wissensformen miteinander kombinieren (TöDTLING und GRILlitsch 2015; GRILLITSCH et al. 2017). Die geographische, demographische und ökonomische Dimension der Peripherisierung kann also um jene der Wissensintensität mit Hilfe der differenzierten Wissensbasen erweitert werden. Es geht folglich nicht nur darum, ob wissensintensive Branchen in einer Region vorhanden sind, sondern auch, welche Art dieses Wissen aufweist (EDER 2019b). Dadurch wird eine sehr detaillierte Betrachtung von Regionen hinsichtlich ihrer Stärken und Schwächen möglich.

\subsection{Innovation in der Peripherie}

Neben einer sinnvollen Abgrenzung stellt sich auch die Frage, ob und wenn ja, weshalb Innovationen in der Peripherie auftreten. Diese Fragestellung wurde in der wirtschaftsgeographischen Forschung lange wenig beachtet, der Fokus lag - und liegt - auf Städten und Clustern und den Vorteilen, die sie unzweifelhaft für Innovationen bieten (SHEARMUR 2012). Dazu zählen beispielsweise eine hochrangige Bildungs- und Forschungsinfrastruktur, ein diverser Arbeitsmarkt und die Verfügbarkeit spezialisierter Dienstleistungen wie etwa Startup-Inkubatoren. Folglich entstehen Durchbrüche in der Grundlagenforschung auch häufig in den urbanen Zentren und sie führen die Patent-Statistiken an. Auch wenn der Forschungsschwerpunkt nach wie vor auf diesen Agglomerationen und den positiven Auswirkungen für ihr Hinterland liegt, beschäftigt sich die Forschung auch zunehmend mit innovativen Peripherien (EDER 2019a).

Dabei stellen sich drei grundlegende Fragen. Erstens: Unter welchen Voraussetzungen ist die Peripherie innovativ? Hier gibt es Indizien, dass Spezialisierung in Nischen vor allem in peripheren Regionen wichtig ist, während Diversität eine zentrale Rolle in Städten spielt (CARAgliU et al. 2016). Der Gestaltungsspielraum der Politik ist allerdings eingeschränkt, da die gleiche Maßnahme - beispielsweise die Förderung von F\&E-Aktivitäten - in verschiedenen Regionen höchst unterschiedliche Resultate erzielen können (CRESCENZI 2005). In Abwesenheit von Agglomerationsvorteilen kommt deshalb vor allem den einzelnen Unternehmen eine Schlüsselrolle zu, die besondere Anstrengungen vollbringen müssen, um gewisse Nachteile zu kompensieren. Mehrere Faktoren spielen hier eine Rolle, wie die strategische Ausrichtung auf Innovation, Unternehmensgröße und -wachstum sowie die Fähigkeit, externes Wissen von außen zu absorbieren und in den eigenen Innovationsprozess zu integrieren (NORTH und SMALLBONE 2000; MCAdam et al. 2004; Copus et al. 2008; MCADAm et al. 2014). Zusätzlich spielen öffentliche Förderungen, eine wirksame Regionalpolitik und Forschungsein- 
richtungen in diesen Regionen eine wichtige Rolle, individuelle Unternehmen hinsichtlich ihrer Innovationstätigkeit und -fähigkeit zu unterstützen (AsHEIM et al. 2019).

Zweitens: Auf welche Art und Weise entstehen Innovationen in der Peripherie? Das Paradigma der Open Innovation, also die aktive und strategische Nutzung von externen Einflüssen im Innovationsprozess, hat in den letzten Jahren an Einfluss gewonnen (Chesbrough 2003). Demnach sind Interaktionen, Spillover-Effekte und externes Wissen zentral. Hier scheinen periphere Regionen im Nachteil, da diese Wissenstransfers und Verflechtungen nicht quasi automatisch durch die höhere Dichte an Unternehmen und Arbeitskräften in einem Stadtraum auftreten. Neben einer starken internen Expertise sind es also Netzwerke zu externen, überregionalen Partnern, die für innovative Unternehmen in peripheren Regionen besonders bedeutend sind (LORENTZEN 2007; Fitjar und RodríGuez-Pose 2011a, 2011b; Dubois 2015, 2016). Es gibt auch Indizien, dass diese Netzwerke häufig formellen Charakter haben und strategisch ausgerichtet sind. Unternehmen gehen hier also gezielt vor, um fehlende Spillover-Effekte zu kompensieren (GRILLITSCH und NiLSSON 2015). Weiters dürften nicht alle innovativen Unternehmen in ländlichen Regionen uneingeschränkt auf das Paradigma der Open Innovation setzen (SHEARMUR 2015).

Und drittens: Welche Innovationen kommen in der Peripherie vor? Zunehmend wird kritisiert, dass die scheinbar geringe Innovationstätigkeit in peripheren Regionen vermutlich auch daran liegt, wie Innovation gemessen wird. Häufig erfolgt dies über Patente, die jedoch nur eine begrenzte Aussagekraft besitzen (DAVIES et al. 2012; SHEARMUR 2015; IsAKSEN und KARLSEN 2016). So sind Prozessinnovationen in Städten häufiger, originäre Innovationen treten aber zu gleichen Teilen in Städten und ländlichen Regionen auf, wenn man Größen- und Brancheneffekte berücksichtigt (LEE und RoDRíGUEZ-Pose 2013). Ebenso sind Innovationen häufiger in traditionellen Sektoren wie der Sachgüterproduktion zu finden, die durch den Fokus auf die Hochtechnologiebranche seltener erforscht werden (ALDERMAN 1998). Da Innovationen in abgelegenen Regionen häufig auch inkrementell sind, gehen einige Autorinnen und Autoren davon aus, dass Unternehmen hier tendenziell eher auf neues Wissen reagieren und keine Innovationsführer sind.

Wie der Blick in die Literatur zeigt, können Unternehmen in peripheren Lagen also zweifellos innovativ sein, sie müssen dafür aber häufig besonderes Engagement zeigen, um diverse Standortnachteile auszugleichen. Dazu zählen die strategische Ausrichtung und Sicherstellung der Einbindung in überregionale Netzwerke. Innovationen treten auch häufiger in Branchen auf, die typisch für diese Regionen sind beziehungsweise durch Unternehmen repräsentiert werden, die schon viele Jahrzehnte am Standort erfolgreich sind. Damit unterscheidet sich auch die Art der Innovationen von jenen in typischen urbanen, wissenschaftsbasierten Innovationsfeldern wie den Lebenswissenschaften. Im letzten Abschnitt des Kapitels wird daher kurz erläutert, wie diese theoretischen Annahmen und der aktuelle Forschungsstand für die vorliegende Studie operationalisiert und konzeptualisiert werden. 


\subsection{Typologie von Peripherisierung und Wissensbasen}

Die Überlegungen hinsichtlich Peripherisierung und regionaler Wissensbasen werden in Abbildung 2.1 konzeptualisiert, um die Vielfalt peripherer aber auch zentraler Regionen zu erfassen und um über die starren Pole Stadt versus Land hinauszugehen (LEICK und LANG 2018; Eder 2019b). Auf der X-Achse wird zunächst der Grad der Peripherisierung gemessen. Periphere Regionen sind demnach solche, die schlecht erreichbar und von demographischer und ökonomischer Stagnation geprägt sind. Demgegenüber stehen zentrale Gebiete, in denen hochrangige Infrastruktur in kurzer Zeit zugänglich ist und Bevölkerung und Wirtschaft wachsen. Dazwischen gibt es viele intermediäre

\section{Abbildung 2.1: Regionstypisierung nach Peripherisierung und regionalen Wissensbasen}

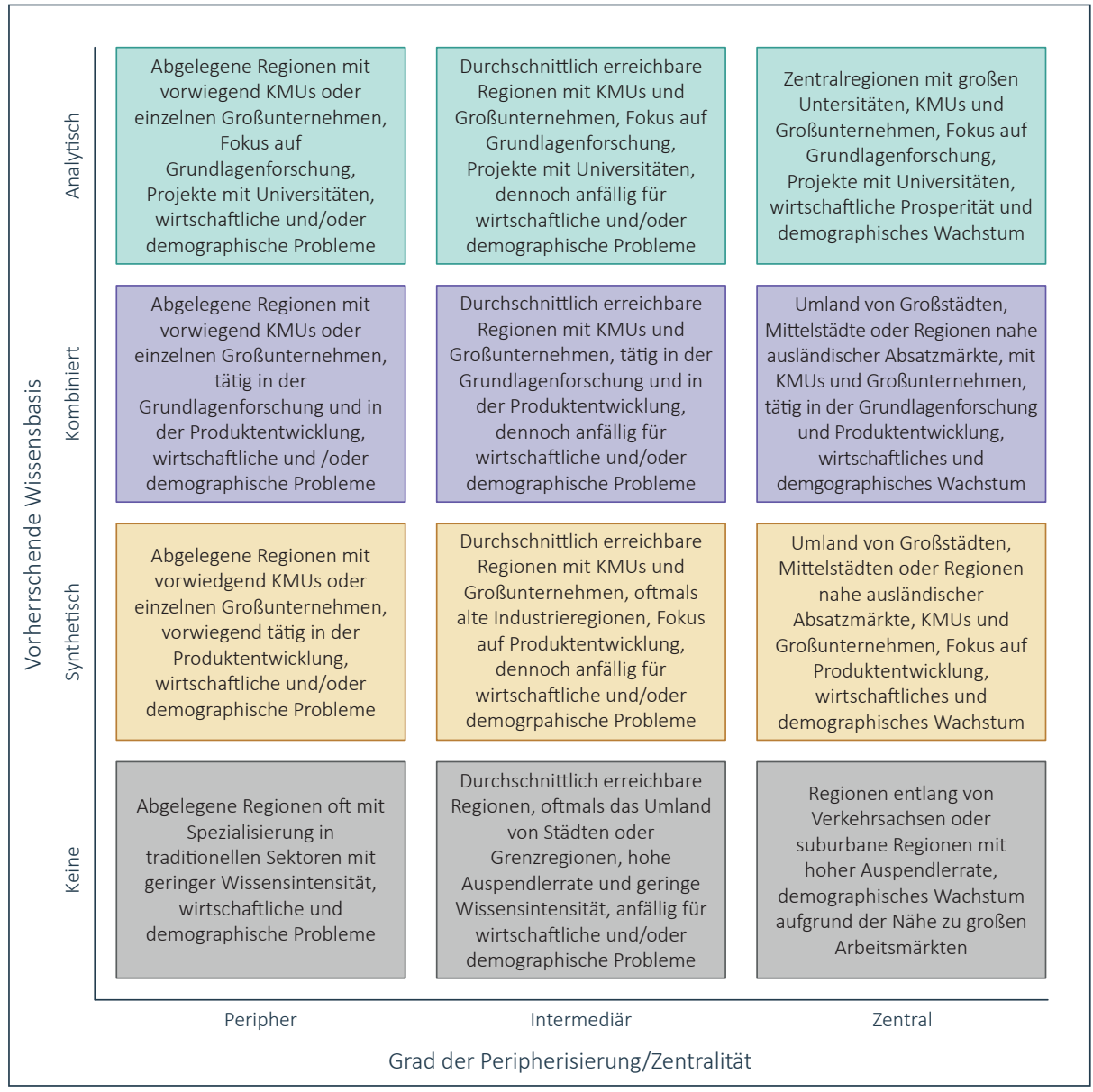


Regionen, die nicht auf all diesen Ebenen zentral beziehungsweise peripher sind, sondern in denen diverse Kombinationen auftreten.

Diese Typologie wird mit der Wissensintensität auf der Y-Achse kombiniert, genauer gesagt mit der vorherrschenden regionalen Wissensbasis. Aus Gründen der Übersichtlichkeit und der Schwierigkeit, die symbolische Wissensbasis quantitativ zu erfassen, werden bei der folgenden Analyse nur die analytische und die synthetische Wissensbasis berücksichtigt. In jenen Regionen, die keine oder nur eine sehr schwache Wissensbasis aufweisen, gibt es in der Regel nur sehr wenige forschungsaktive und damit innovative Unternehmen. Eine vorwiegend synthetische Wissensbasis findet sich in solchen Gebieten, in denen Unternehmen im Innovationsprozess verstärkt auf Qualifikationen setzen, die am Arbeitsplatz erlernt werden und die auf experimentelle Entwicklung fokussieren. Im Gegensatz dazu gibt es Regionen, die ihre Stärken in der analytischen Wissensbasis besitzen. Unternehmen in diesen Regionen haben in ihren Forschungsabteilungen viele Beschäftigte mit Hochschulbildung, arbeiten vermehrt mit Universitäten zusammen und sind in der Grundlagenforschung aktiv. Die letzte Kategorie bilden jene Gebiete, die sowohl hinsichtlich der synthetischen als auch der analytischen Wissensbasis überdurchschnittlich abschneiden. Dabei ist davon auszugehen, dass jene Regionen die wettbewerbsfähigsten sind, da eine Kombination der Wissensbasen in der Regel zum höchsten Innovationsoutput führt (GRILLITSCH et al. 2015; GriLLITSCH et al. 2017).

Dieses Framework geht folglich davon aus, dass nicht alle zentralen Regionen auch die besten Voraussetzungen für wissensbasierte Innovationen mitbringen, aber auch nicht alle peripheren Gebiete keine dieser Vorbedingungen besitzen. Kategorien wie Stadt und Land sind hinsichtlich ihrer Innovationsfähigkeit also diversifizierter zu betrachten.

Für den zweiten, qualitativen Teil der Analyse ist es notwendig, auf die Strategien innovativer Unternehmen in abgelegenen Regionen einzugehen, mit denen Nachteile der Heimatregion kompensiert beziehungsweise Vorteile genutzt werden (siehe Tabelle 2.1). Einerseits ist davon auszugehen, dass die regionalen Rahmenbedingungen eine Rolle spielen. Bietet eine periphere Region kaum Innovationsvorteile, so wird sich ein Unternehmen vorwiegend auf Strategien beschränken, um Standortnachteile auszugleichen. Zu diesen Kompensationsstrategien zählen etwa der Ausbau überregionaler Netzwerke, die Teilnahme an temporären Clustern (beispielsweise Messen oder Konferenzen) oder virtuellen Wissensquellen (beispielsweise Webinare) (EDER und TRIPPL 2019).

Besitzt eine Peripherie andererseits jedoch auch Innovationsvorteile, dürfte ein Unternehmen auch Inwertsetzungsstrategien anwenden. So ist davon auszugehen, dass ein schützendes Umfeld einen Vorteil darstellt. Die Loyalität der Belegschaft ist in ländlichen Regionen häufig sehr hoch, somit werden auch unerwünschte Wissensabflüsse aus dem Unternehmen minimiert. Innovative Unternehmen haben in peripheren Regionen auch häufig eine Sonderstellung, wodurch sie einen besonderen Spielraum in der Region genießen. Dieser zeigt sich etwa in der zielgenauen Zusammenarbeit mit regio- 
nalen Bildungseinrichtungen oder einer großen Unterstützung durch die Politik. Und schließlich spielen auch finanzielle Anreize wie ein niedrigeres Lohnniveau, geringe Grundstückspreise oder öffentliche Förderungen eine Rolle (EDER und TRIPPL 2019).

\section{Tabelle 2.1: Anwendung von Kompensations- und Nutzungsstrategien in Abhängigeit regionaler Rahmenbedingungen}

\begin{tabular}{llll} 
& & \multicolumn{2}{c}{ Innovationsbarrieren } \\
\cline { 3 - 4 } & Keine/Wenige & \multicolumn{1}{c}{ Keine/Wenige } & \multicolumn{1}{c}{ Zahlreiche } \\
\hline $\begin{array}{c}\text { Selektive Auswahl einiger } \\
\text { weniger Kompensations- } \\
\text { strategien }\end{array}$ & $\begin{array}{l}\text { Auswahl zahlreicher Kom- } \\
\text { pensationsstrategien } \\
\text { vorteile }\end{array}$ & $\begin{array}{l}\text { Selektive Auswahl einiger } \\
\text { weniger Kompensations- } \\
\text { und zahlreicher Nutzungs- } \\
\text { strategien }\end{array}$ & $\begin{array}{l}\text { Auswahl zahlreicher Kom- } \\
\text { pensations- und Nutzungs- } \\
\text { strategien }\end{array}$ \\
\hline
\end{tabular}

Neben diesen regionalen Rahmenbedingungen sind aber noch firmenspezifische Faktoren von Bedeutung, die einen Einfluss auf die Auswahl der Kompensations- und Inwertsetzungsstrategien haben. Die Unternehmensgröße ist bedeutend, da mit ihr in der Regel auch die Ressourcen steigen, die ein Unternehmen beispielsweise für die Aufrechterhaltung externer Wissensnetzwerke zur Verfügung hat. Auch der Gestaltungsspielraum am Standort steigt mir der Größe. Zusätzlich sind die Branchenzugehörigkeit oder die Unternehmenskultur in diesem Zusammenhang zu berücksichtigen. Manche Nach- oder Vorteile mögen für eine Branche relevant sein, für eine andere weniger. Und einige Firmen dürften sich stärker auf Verschwiegenheit verlassen, andere das Paradigma der Open Innovation engagierter umsetzen. Folglich ist ein sehr diverses Bild der Kompensations- und Inwertsetzungsstrategien zu erwarten.

Der nächste Abschnitt geht auf die Methodik ein, mit der einerseits quantitativ die Rahmenbedingungen für Innovation in Österreich auf Ebene der Bezirke gemessen werden andererseits wird die Vorgehensweise diskutiert, mit der 20 Unternehmen in Österreich zu diesen Themen befragt wurden. 